\title{
ORIGINAL ARTICLE Reactivation of androgen receptor-regulated lipid biosynthesis drives the progression of castration-resistant prostate cancer
}

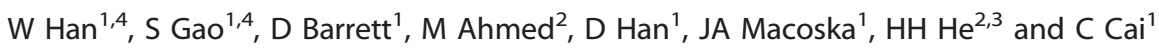

\begin{abstract}
Androgen receptor (AR) is a transcriptional activator that, in prostate cells, stimulates gene expression required for various cellular functions, including metabolisms and proliferation. AR signaling is also essential for the development of hormone-dependent prostate cancer (PCa) and its activity can be blocked by androgen-deprivation therapies (ADTs). Although PCa patients initially respond well to ADTs, the cancer inevitably relapses and progresses to lethal castration-resistant prostate cancer (CRPC). Although AR activity is generally restored in CRPC despite the castrate level of androgens, it is unclear whether AR signaling is significantly reprogrammed. In this study, we examined the AR cistrome in a PCa cell line-derived CRPC model using integrated bioinformatical analyses. Significantly, we found that the AR cistrome is largely retained in the CRPC stage. In particular, AR-mediated lipid biosynthesis is highly conserved and reactivated during the progression to CRPC, and increased level of lipid synthesis is associated with poor prognosis. The restoration of lipid biosynthetic pathways is partially due to the increased expression of AR splice variants. Blocking lipid/cholesterol synthesis in AR variants-expressing CRPC cell line and xenograft models markedly reduces tumor growth through inhibition of mTOR pathway. Silencing the expression of a fatty acid elongase, ELOVL7, also leads to the regression of CRPC xenograft tumors. These results demonstrate the importance of reactivation of AR-regulated lipid biosynthetic pathways in driving CRPC progression, and suggest that ADTs may be therapeutically enhanced by blocking lipid biosynthetic pathways.
\end{abstract}

Oncogene (2018) 37, 710-721; doi:10.1038/onc.2017.385; published online 23 October 2017

\section{INTRODUCTION}

Androgen receptor (AR) is a well-established transcription factor that is essential for normal prostate development and function. In prostate cancer (PCa), AR also plays a pivotal role in driving tumor growth, and patients with metastatic PCa can be treated with surgical or medical castration (androgen-deprivation therapy, ADT). However, most patients relapse within several years despite castrate androgen levels, and this more aggressive form of $\mathrm{PCa}$ is called castration-resistant PCa (CRPC). ${ }^{1,2}$ The recurrence of the cancer is largely due to residual androgens that contribute to persistent AR activity and, therefore, many patients with CRPC respond to therapies that further suppress androgen synthesis or to more potent AR antagonists..$^{3-5}$ However, patients still generally relapse within 1-2 years and high levels of $A R$ expression and rising levels of AR-regulated genes in these patients suggest that AR activity is once again being restored. Therefore, there continues to be a pressing need for improving AR-targeted therapies in PCa.

It is now clear that the AR cistrome is significantly changed during the initial development of PCa. In PCa cells, AR has gained transcriptional control of many new oncogenic targets, including Ets family genes (through chromosomal alterations) and SOX9, whose expression may also alter AR-binding patterns in $\mathrm{PCa}$ cells. $^{6-9}$ In addition, AR chromatin binding can be also reprogrammed through altered expression of various pioneer factors, coactivators/corepressors, and epigenetic regulators. ${ }^{10} \mathrm{~A}$ recent AR genome-wide binding study on radical prostatectomy tumors and benign epithelium strongly suggests that $A R$ has undergone intensive reprogramming in cooperation with FOXA1 and HOXB13 in prostate tumorigenesis. ${ }^{11} \mathrm{AR}$ reprogramming has also been reported in CRPC models or samples. ${ }^{12,13}$ One potential mechanism for reprogramming the AR cistrome in CRPC might be through the increased expression of certain AR splice variants (AR-Vs), ${ }^{14-19}$ which can be constitutively activated and may bind to enhancer sites that are distinct from full-length AR. However, recent studies on AR-Vs indicate that many of them may heterodimerize with full-length $A R$ and thus bind to the same sites as full-length $A R{ }^{18}$ Nonetheless, additional in vivo models need to be developed for assessing reprogramming of AR cistrome in CRPC.

In this study, we have used a VCaP (an androgen-sensitive PCa cell line) derived xenograft model ${ }^{20-23}$ to determine whether AR signaling is substantially reprogrammed during the progression to CRPC. VCaP-derived xenografts are initially androgen dependent and respond to castration. However, the xenograft tumors eventually relapse after castration with elevated expression of AR, AR-Vs, and steroid synthetic genes, which closely mimic the characteristics of human CRPC. ${ }^{20-23}$ We extracted the cells from the relapsed xenograft tumors (called VCS2 cells) and subsequently identified AR cistrome in these cells. Significantly, ARbinding sites largely overlap between VCS2 and parental VCaP cells, and the AR-regulated gene signature is highly maintained. Importantly, we show that genes mediating lipid biosynthesis are the major targets of AR in VCaP cells, and that this transcription program is highly conserved and reactivated in VCS2 cells. Since

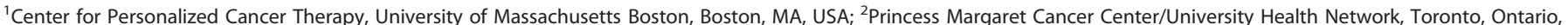

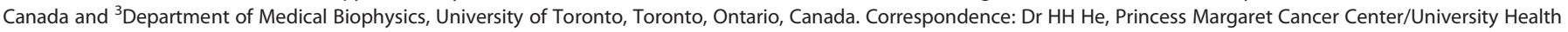

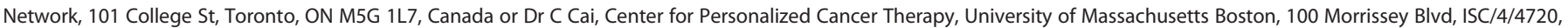
Boston, MA 02125, USA.

E-mail: hansenhe@uhnresearch.ca or changmeng.cai@umb.edu

${ }^{4}$ These authors contribute equally to this work.

Received 6 February 2017; revised 30 July 2017; accepted 12 September 2017; published online 23 October 2017 
the expression of V7 variant (AR-V7, also termed $A R 3)$ is substantially increased in our CRPC models, we then examined the influence of AR-V7 overexpression on those lipid synthesis genes. Using CWR22-RV1 cell line model, we showed that endogenous AR-V7 strongly binds to the same binding sites within lipid synthesis genes as full-length AR does and contributes to their expression. Furthermore, we also demonstrated that inhibition of lipid/cholesterol biosynthesis significantly reduced the growth of castration-resistant xenograft tumors derived from CWR22-RV1 cells. This growth-inhibitory effect of lipid synthesis inhibitor is not through blocking the androgen synthesis pathway but through inactivation of mTOR. Silencing the expression of an AR-regulated fatty acid elongase, ELOVL7, also significantly decreased the growth of CWR22-RV1 xenograft tumors. Overall, this study provides a strong rationale for blocking lipid synthesis in combination with more intensive ADTs for treating CRPC patients expressing $A R-V s$ at risk for tumor recurrence and relapse.

\section{RESULTS}

Global analysis of the AR cistrome in a VCaP-derived CRPC model We have previously established a CRPC xenograft model derived from androgen-sensitive VCaP PCa cells and this model closely mimics the progression of CRPC in human patients with increased androgen synthesis and elevated level of full-length $A R$ and $A R$ splice variants. ${ }^{20-23}$ We then extracted the cells from the recurrent xenograft tumors and only allowed minimum passages in tissue culture to preserve the characteristics of CRPC. Using this method, we have established VCS2 cells for a short period of time, which express higher levels of $A R$ mRNA and protein as well as androgen synthesis genes than the parental VCaP cells. ${ }^{21,22}$ To determine whether AR signaling is significantly altered in CRPC, we carried out AR ChIP-seq analyses (antibody against AR N-terminal domain) in VCaP/VCS2 cells treated for $4 \mathrm{~h}$ of $10 \mathrm{~nm}$ DHT (database: GSE32345). This analysis yielded a comparable number of binding peaks between the two cell lines (11850 in VCS2 versus 12210 in VCaP) and a marked overlap (8872) using a stringent threshold $\left(P<10^{-15}\right)$ (Figure 1a). As expected, the overlapping AR-binding sites were enriched for consensus AR/GR/PR and Forkhead box protein binding motifs (Figure $1 \mathrm{~b}$ ). An analysis of non-overlapping binding sites similarly yielded enrichment in AR/GR/PR-binding motifs (Figures $1 \mathrm{c}$ and $\mathrm{d}$ ). While these sites were non-overlapping, which is based on peak calling using a stringent threshold $\left(P<10^{-15}\right)$, AR-binding intensities were lower than at overlapping sites and weaker but detectable binding could generally be found in both cell lines, indicating that most of these sites may be preferred in VCaP or VCS2, but are not specific.

We next examined AR-binding sites close to genes ( $20 \mathrm{~kb}$ from transcription start site, TSS) that were activated by $24 \mathrm{~h}$ DHT treatment (two-fold cutoff) in VCaP and VCS2 cells (database: GSE32344). We found enrichment for AR-binding sites in association with androgen-stimulated genes (Figures $2 a$ and $b$, left panel). Gene Ontology analysis of androgen-stimulated genes with associated AR-binding sites in VCaP and VCS2 cells showed similar enrichment for pathways, particularly lipid and sterol metabolism, consistent with previous findings indicating that a major function of AR is to regulate cellular metabolic pathways (Figures $2 a$ and $b$, right panel). ${ }^{22,24,25}$ Using a less stringent threshold $\left(P<10^{-5}\right)$ to identify AR-binding sites within $20 \mathrm{~kb}$ from TSS of these AR-activated genes, we identified 32 androgenstimulated lipid synthesis genes in VCS2 cells (Table 1). As expected, all the genes, except for CYP1A1, were also androgen activated in VCaP cells. These genes mediate variety of lipid biosynthesis pathways, including fatty acid, cholesterol, and sterol synthesis, and some of them have been previously shown to be associated with PCa development ${ }^{26-29}$ (Figure 2c).
Although AR-binding is largely conserved in VCS2 cells, the small subset of VCS2-prefered sites (2978) may result in the differential regulation of distinct pathways involved in CRPC progression. Therefore, we also explored the AR binding and the gene expression data to determine whether AR was regulating a functionally distinct set of genes in VCS2 cells. However, this analysis yielded very few genes that were directly activated by AR only in VCS2 cells (Figure 2d), and these genes could not be linked to any specific pathways. It is notable that these non-overlapping sites identified based on a stringent threshold have lower intensities than the overlapping sites, and have weaker but detectable binding in both cell lines. These findings suggest that the AR cistrome is largely conserved during CRPC progression, and that activation of lipid biosynthesis pathways is a major function of AR signaling in PCa progression.

Since the above work was performed in cell line-derived models, we next determined whether these AR-binding sites adjacent to lipid synthesis genes could be found in normal prostate and PCa cells from human patient tissues using a public ChIP-seq database. ${ }^{11}$ In those samples, $>60 \%$ of the lipid synthesis genes consistently harbor adjacent AR-binding sites at the same locations as in DHT treated VCaP and VCS2 cells (Figure 2e), supporting that PCa cells may have preserved this ARbinding signature during disease initiation and progression.

\section{AR-mediated lipid synthesis is restored in CRPC}

We then selected a few AR-activated lipid synthesis genes for further validation. These genes included long-chain acyl-CoA Synthetase 3 (ACSL3), Membrane-bound O-acyltransferase domain containing protein 2 (MBOAT2), and ELOVL fatty acid elongase 5 and 7 (ELOVL5/7). Consistent to the gene profiling data, the expression of these genes were androgen stimulated in VCaP and VCS2 cells as well as in LNCaP cells (another widely-used androgen-sensitive PCa cell line) (Figure 3a). ChIP-qPCR also confirmed androgen-induced AR occupancy at the sites identified by ChIP-seq for each gene in VCaP or LNCaP cells, and treating enzalutamide (a potent AR antagonist) impaired AR-binding to these sites (Figure 3b).

We next sought to determine whether the expression of lipid synthesis genes is restored in CRPC in vivo. To accomplish this, tumor biopsies were obtained from VCaP xenografts prior to castration or at relapse and examined for expression of lipid synthesis genes. As seen in Figure 3c, the expression of each gene examined was generally restored in a similar degree with ARregulated PSA and TMPRSS2. We further examined the restoration of lipid synthesis genes expression levels in tumor samples from patients with hormone-dependent primary PCa or bone metastatic CRPC. ${ }^{30}$ In those patient biopsies, the AR activity in CRPC was partially restored as indicated by AR-regulated expression of PSA and TMPRSS2 (Figure 3d). The expression of lipid synthesis genes was generally restored in a similar degree (if not stronger) to the partial reactivation of classic AR-regulated genes (Figure 3d). Finally, we assessed whether the expression of lipid synthesis genes correlates with patient prognosis in clinical data sets and found that a higher level of expression is consistently associated with poor patient outcome and disease recurrence in all three cohorts that were examined ${ }^{31-33}$ (Figure 3e), indicating the important role of lipid biosynthesis in driving PCa progression.

The expression of AR splice variants promotes the reactivation of lipid synthesis in CRPC cells

One mechanism that potentially contributes to the reactivation of AR signaling in CRPC is expression of the constitutively active $A R$ splice variants. ${ }^{34}$ Several $A R$ splice variants, including $A R-V 1, V 7$, and $V 8-11$, were previously detected in VCaP models and the expression of $A R-V 7$ was shown to be increased by androgen withdraw. $^{23,34,35}$ Consistently, we observed markedly increased 


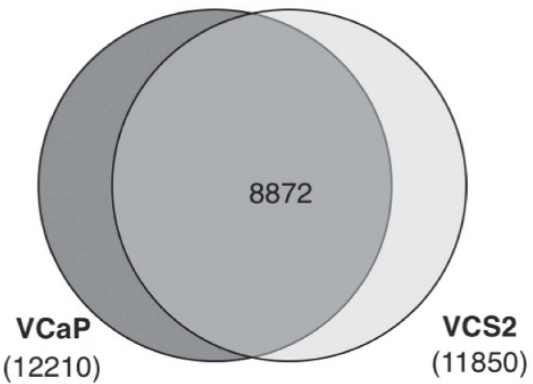

b

VCaP/VCS2 overlapping sites $(8,872)$
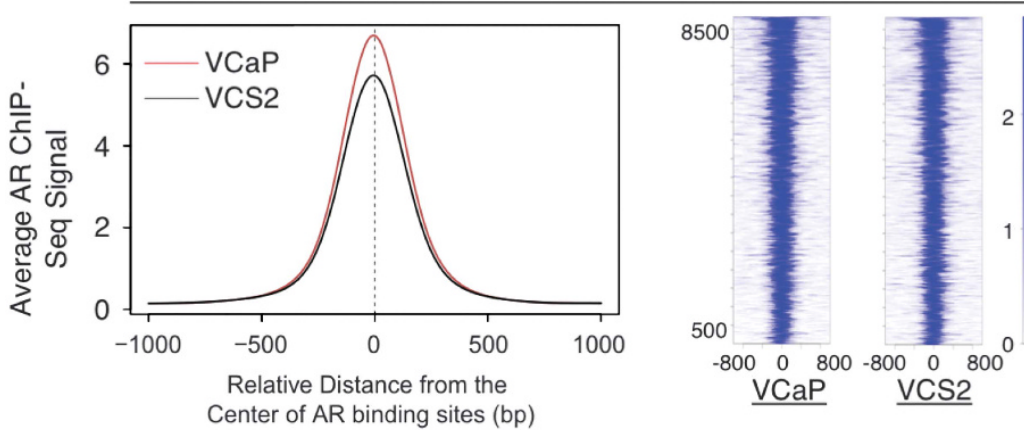

$\begin{array}{lll}\text { ID } & \text { Factors } & \text { Consensus } \\ \text { M00957 } & \text { PR } & \text { 15TGTTC7 } \\ \text { M00481 } & \text { AR } & \text { 1G2C5GT1C1 } \\ \text { M00955 } & \text { GR } & \text { 15TGTTC7 } \\ \text { M00956 } & \text { AR } & \text { 15TGTTC7 } \\ \text { M00953 } & \text { AR } & \text { 15TGTTC7 } \\ \text { M00954 } & \text { PR } & \text { 15TGTTC7 } \\ \text { M00447 } & \text { AR } & \text { AG8GT1C1 } \\ \text { M00205 } & \text { GR } & \text { 3A6GT1CT1 } \\ \text { M00960 } & \text { PR, GR } & \text { GWDAGRACAB } \\ \text { M00921 } & \text { GR } & \text { AGRACASW } \\ \text { M00289 } & \text { HFH3 (FOXI1) } & \text { 1A3AAA1A3 } \\ \text { M00294 } & \text { HFH8 (FOXF1A) 3T1TTT1T3 } \\ \text { M00724 } & \text { HNF3alpha } & \text { HWRARYAAAYA } \\ \text { M00292 } & \text { Freac-4 } & \text { 6T1AACA4 } \\ \text { M00290 } & \text { Freac-2 } & \text { 6TAAA1A4 }\end{array}$

C

VCaP preferred sites $(3,338)$
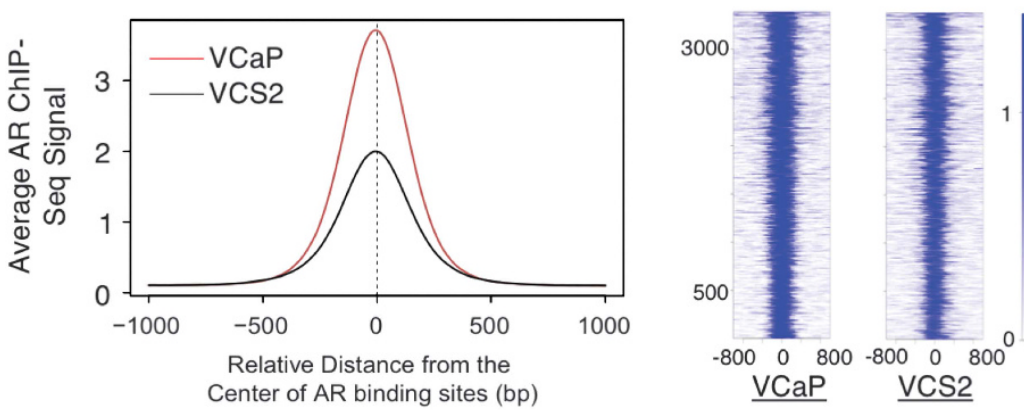

$\begin{array}{lll}\text { ID } & \text { Factors } & \text { Consensus } \\ \text { M00955 } & \text { GR } & \text { 6AGAAC16 } \\ \text { M00957 } & \text { PR } & \text { 6AGAAC16 } \\ \text { M00954 } & \text { PR } & \text { 6AGAAC16 } \\ \text { M00956 } & \text { AR } & \text { 6AGAAC16 } \\ \text { M00953 } & \text { AR } & \text { 6AGAAC16 } \\ \text { M00380 } & \text { Pax-4 } & \text { 1G2T20TTT2 } \\ \text { M00481 } & \text { AR } & \text { 1G1AC5G2C1 } \\ \text { M00724 } & \text { HNF3alpha } & \text { HWRARYAAAYA } \\ \text { M00143 } & \text { Pax-5 } & \text { 1C6CA2G2G12 } \\ \text { M00289 } & \text { HFH3 (FOXI1) } & \text { 3T1TT1T3 } \\ \text { M00791 } & \text { HNF3 } & \text { 5YAAA1A2 } \\ \text { M00921 } & \text { GR } & \text { AGRACASW } \\ \text { M00290 } & \text { Freac-2 } & \text { 4T1TTTA6 } \\ \text { M00294 } & \text { HFH8 (FOXF1A) 3A1AAA1A3 } \\ \text { M01012 } & \text { HNF3 } & \text { 5A2AAA1A5 }\end{array}$

d

VCS2 preferred sites $(2,978)$
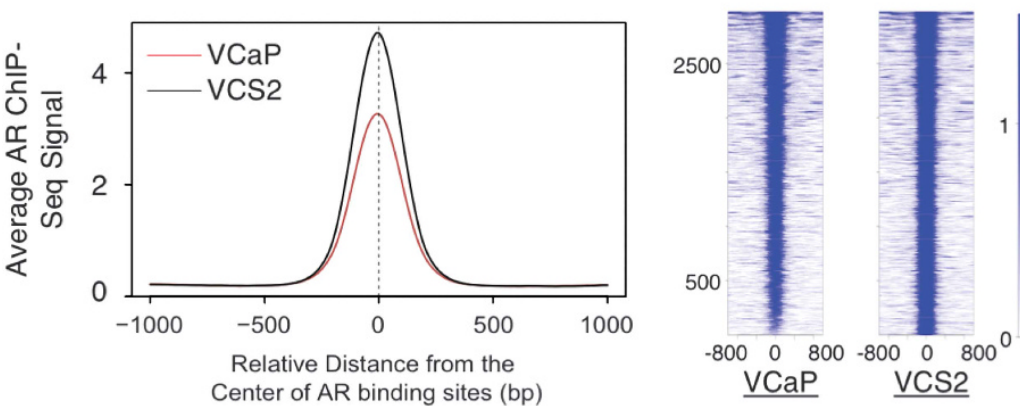

$\begin{array}{lll}\text { ID } & \text { Factors } & \text { Consensus } \\ \text { M00955 } & \text { GR } & \text { 6AGAAC16 } \\ \text { M00957 } & \text { PR } & \text { 6AGAAC16 } \\ \text { M00956 AR } & \text { 6AGAAC16 } \\ \text { M00954 PR } & \text { 6AGAAC16 } \\ \text { M00481 } & \text { AR } & \text { 1G1AC5G2C1 } \\ \text { M00953 } & \text { AR } & \text { 6AGAAC16 } \\ \text { M00921 GR } & \text { WSTGTYCT } \\ \text { M00960 PR, GR } & \text { VTGTYCTHWC } \\ \text { M00380 Pax-4 } & \text { 1G2T20TT2 } \\ \text { M00447 } & \text { AR } & \text { AG1AC8C1 } \\ \text { M00192 } & \text { GR } & \text { 11TGT1CT2 } \\ \text { M00205 GR } & \text { 1AG1AC6T3 } \\ \text { M00143 Pax-5 } & \text { 1C6CA2G2G12 } \\ \text { M01036 COUPTF } & \text { 6G3A2G2CA5 } \\ \text { M01087 CEBP } & \text { 5T1TG2M2W8 } \\ & & \end{array}$

Figure 1. AR cistrome is retained in VCS2 cells versus VCaP cells. (a) VCaP and VCS2 cells in androgen-deprived medium were treated with $10 \mathrm{~nm}$ of DHT for $4 \mathrm{~h}$, followed by AR ChIP-Seq. High confident peaks were identified using MACS $\left(P\right.$-value $\left.<10^{-15}\right)$. Overlap of AR ChIP-seq between VCaP and VCS2 cells was shown. (b-d) Average AR-binding intensity histogram (left panel) or heatmap (middle panel), or most enriched binding motifs (right panel) in (b) overlapping sites versus (c) VCaP preferred or (d) VCS2 preferred sites.

expression of $A R-V 7$ splice variant in VCaP-derived CRPC xenograft tumors (see Figure $3 c$ ) and in VCS2 cells (Figure $4 a$, left panel). The ratio of $A R-V 7$ to full-length $A R$ was also increased in VCS2 cells compared with VCaP cells (Figure $4 a$, right panel). These observations are also consistent with previous reports showing that ADT can increase the expression of AR variants. ${ }^{19,23,36}$ Using an AR-V7-specific antibody (against the region of cryptic exon 3), we have detected significant binding of AR-V7 at the previously identified AR-binding sites adjacent to lipid synthesis genes (Figure 4b). Due to the limited availability of VCS2 cells, we chose another AR-Vs-expressing CRPC cell line, CWR22-RV1 cells, to further examine the contribution of AR splice variants to the reactivation of lipid biosynthesis. In CWR22-RV1 cells, multiple forms of $A R-V s$, including $V 1-7$, are highly expressed due to the AR 
a VCaP

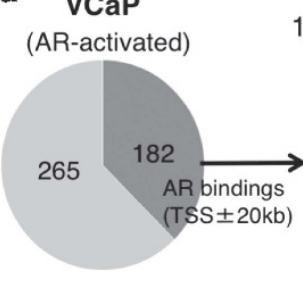

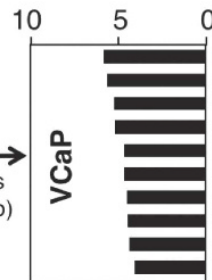

$0-\log _{10}(p-$ Value $)$

lipid biosynthetic process sterol metabolic process cholesterol metabolic process sterol biosynthetic process response to extracellular stimulus Desponse exranic substamus response organic substance cholesterol biosynthetic process steroid biosynthetic proces steroid metabolic process response to drug

b VCS2

(AR-activated)
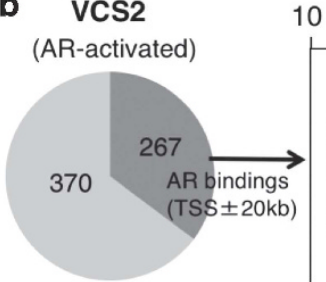

$0-\log _{10}(p-$ Value $)$

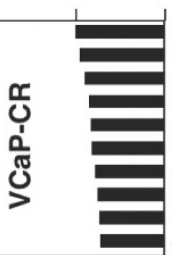

lipid biosynthetic process esponse to extracellular stimulus sterol metabolic process sterol biosynthetic process response to organic substance steroid biosynthetic process cholesterol metabolic process steroid metabolic process response to inorganic substance cholesterol biosynthetic process

d

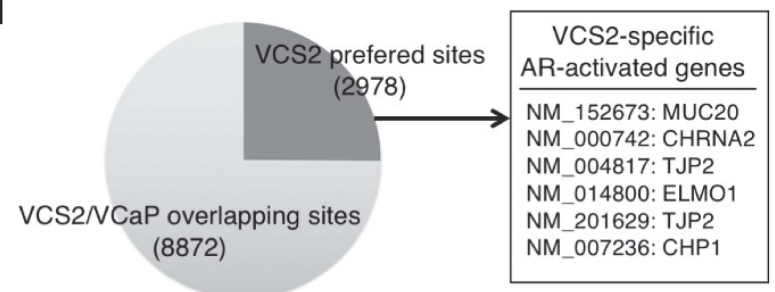

c

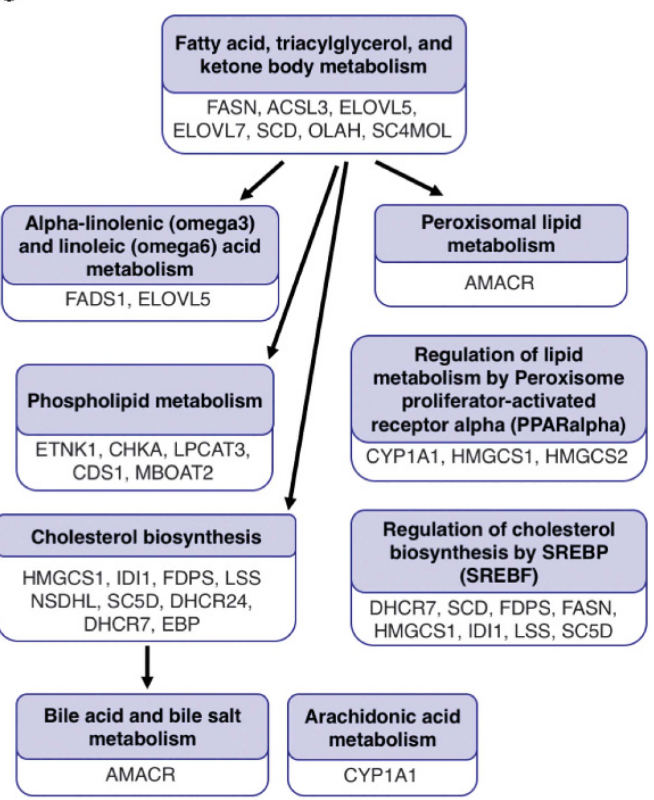

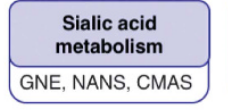

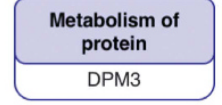

e

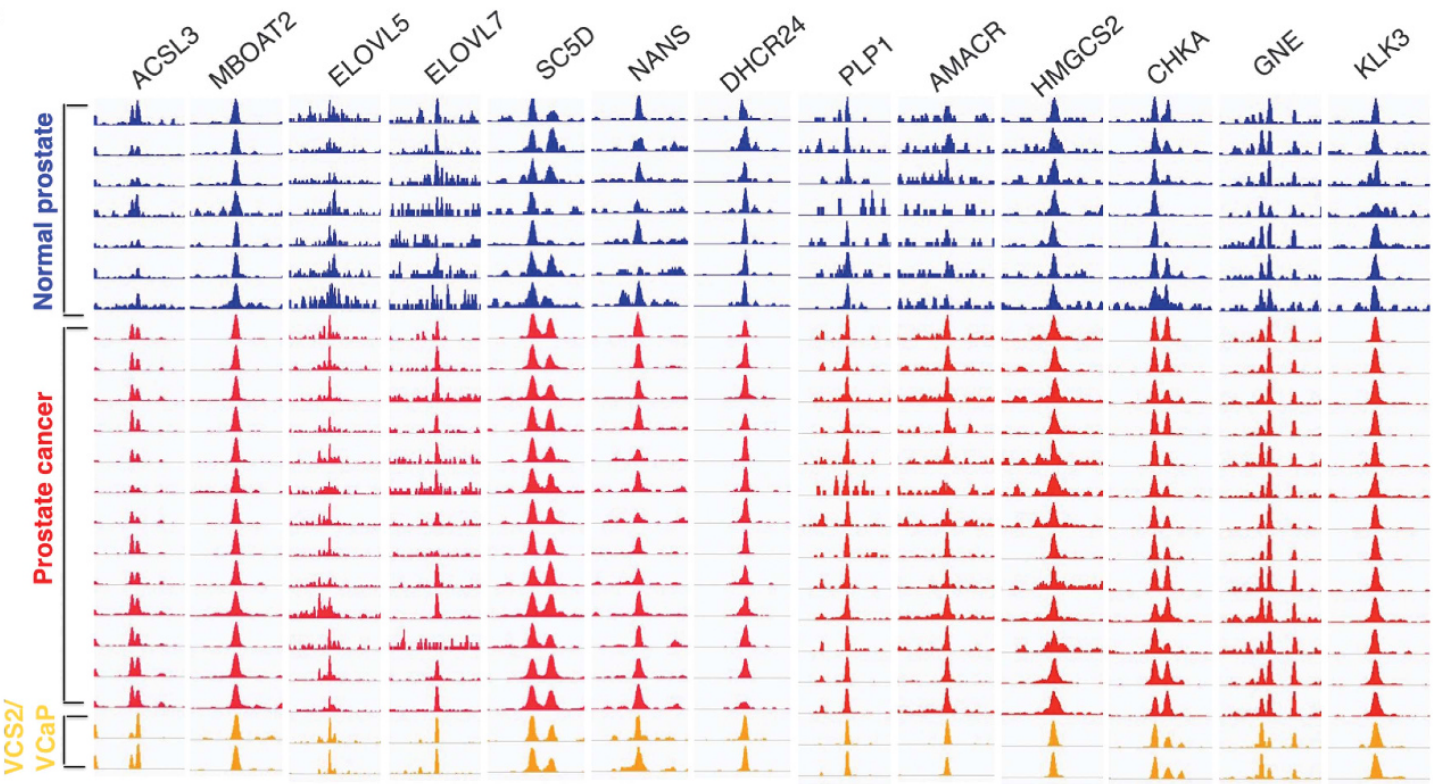

Figure 2. Lipid biosynthesis is the major target of AR in VCaP and VCS2 cells. (a, b) Integrated analyses of AR ChIP-seq and AR-regulated gene expression in VCaP and VCS2 cells (10 nM DHT for $24 \mathrm{~h}$ ). Gene Ontology analysis of direct AR-activated genes in (a) VCaP cells and (b) VCS2 cells. This analysis is based on AR-binding sites within $20 \mathrm{~kb}$ of transcriptional start sites and two-fold cutoff for change of expression. (c) The list of identified AR-activated genes mediating variety of lipid synthetic pathways. (d) The list of VCS2-specific AR-regulated genes with ARbinding. (e) AR ChIP-seq signal of a panel of identified AR-binding sites within 7 normal prostate samples and 13 PCa samples (database: GSE70079)

intragenic rearrangement, ${ }^{37}$ but only $A R-V 1$ and $V 7$ are commonly found in clinical samples. ${ }^{16}$ Similar to VCaP cells, CWR22-RV1 cells exhibited androgen regulation of lipid synthesis genes (Figure 4c) and AR-V7 binds to these AR-binding sites under hormonedepleted condition (Figure 4d). As previous reports indicated that AR-V7 may heterodimerize with full-length AR (AR-FL) and thus enhance its activity in CRPC cells, we examined this activity in response to androgen stimulation and antagonist treatment. Similar to the binding of total AR (antibody against N-terminal region), the binding of $\mathrm{AR}-\mathrm{V} 7$ was enhanced by $\mathrm{DHT}$ and this increase was blocked by enzalutamide (Figure 4e), indicating that AR-V7 cooperates with AR-FL in presence of androgens. Interestingly, the AR-V7 binding was not androgen stimulated in VCaP cells (see Figure 4b). This difference of AR-V7 binding may reflect much higher expression levels of AR-FL in VCaP cells due to gene amplification, or other mechanisms. Nevertheless, we next determined whether AR-V7 expression contributes to the transcription of the lipid synthesis genes. As seen in Figures $4 f$ and $g$, 
Table 1. List of lipid biosynthetic genes that are androgen-stimulated and harbor adjacent AR-binding sites

\begin{tabular}{|c|c|c|c|c|}
\hline Assesion & Gene ID & $\begin{array}{l}\text { VCS2 (fold- } \\
\text { change) }\end{array}$ & $\begin{array}{l}\text { VCaP (fold- } \\
\text { change) }\end{array}$ & $\begin{array}{c}\text { AR-binding (VCS2 } \\
\text { and VCaP) }\end{array}$ \\
\hline NM_000533 & PLP1 & 7.1 & 5.1 & ++ \\
\hline NM_014324 & AMACR & 5.0 & 1.9 & ++ \\
\hline NM_203372 & ACSL3 & 4.4 & 4.2 & ++ \\
\hline NM_006745 & SC4MOL & 4.3 & 2.9 & + \\
\hline NM_005518 & HMGCS2 & 4.1 & 2.2 & ++ \\
\hline NM_004508 & IDI1 & 3.9 & 2.7 & + \\
\hline NM_004104 & FASN & 3.7 & 1.4 & + \\
\hline NM_005063 & SCD & 3.6 & 1.5 & + \\
\hline NM_024930 & ELOVL7 & 3.5 & 2.0 & ++ \\
\hline NM_138799 & MBOAT2 & 3.4 & 2.5 & ++ \\
\hline NM_005476 & GNE & 3.4 & 3.6 & ++ \\
\hline NM_000499 & CYP1A1 & 3.2 & 0.6 & + \\
\hline NM_006918 & SC5D & 3.2 & 2.2 & ++ \\
\hline NM_015922 & NSDHL & 3.1 & 2.5 & ++ \\
\hline NM_018638 & ETNK1 & 3.1 & 2.0 & + \\
\hline NM_002130 & HMGCS1 & 2.9 & 1.1 & + \\
\hline NM_006579 & EBP & 2.9 & 2.1 & N/A \\
\hline NM_013402 & FADS1 & 2.6 & 1.6 & + \\
\hline NM_018677 & ACSS2 & 2.5 & 1.5 & + \\
\hline NM_002340 & LSS & 2.4 & 1.4 & ++ \\
\hline NM_018946 & NANS & 2.4 & 2.7 & + \\
\hline NM_002004 & FDPS & 2.4 & 1.5 & + \\
\hline NM_018324 & OLAH & 2.3 & 1.2 & + \\
\hline NM_001277 & CHKA & 2.2 & 1.1 & ++ \\
\hline NM_001360 & DHCR7 & 2.2 & 1.7 & ++ \\
\hline NM_014762 & DHCR24 & 2.2 & 2.9 & ++ \\
\hline NM_005768 & LPCAT3 & 2.1 & 2.0 & + \\
\hline NM_018973 & DPM3 & 2.1 & 1.6 & $\mathrm{~N} / \mathrm{A}$ \\
\hline NM_018686 & CMAS & 2.1 & 1.3 & ++ \\
\hline NM_001263 & CDS1 & 2.0 & 2.1 & + \\
\hline NM_021814 & ELOVL5 & 2.0 & 1.9 & + \\
\hline NM_021727 & FADS3 & 2.0 & 1.5 & N/A \\
\hline \multicolumn{5}{|c|}{$\begin{array}{l}\text { Integrated analysis of ChIP-seq of AR and gene expression in VCaP and } \\
\text { VCS2 cells identified } 32 \text { direct AR-activated genes that mediate lipic } \\
\text { synthesis pathways. ('++' indicates the AR-binding sites identified using } P \\
\text { value }<10^{-15} \text {, while ' }+ \text { ' indicates the AR-binding sites using } P \\
\text { value }<10^{-5} \text { ). }\end{array}$} \\
\hline
\end{tabular}

the expression of AR-V7 or AR-FL can be specifically silenced by siRNA against cryptic exon 3 (siCE3) or against exon 7 (siE7), respectively. Interestingly, silencing AR-FL resulted in completely block of androgen-induced (low to intermediate level of DHT) expression of lipid biosynthesis genes, while it did not significantly repress the basal expression of these genes (Figure 4h). In contrast, silencing AR-V7 consistently decreased the basal expression of all three lipid synthesis genes ( 30-40\%), but had less effect on androgen-induced expression, suggesting that AR-V7 may function independently to increase the basal expression of these genes. Overall, these results support the role of increased expression of $A R-V 7$ as an important mechanism that contributes to the reactivation of lipid synthesis in CRPC.

\section{Blocking the lipid biosynthesis suppresses CRPC tumor growth} We next sought to determine the functional importance of lipid/ sterol biosynthesis in driving CRPC progression. As seen in Figure $5 \mathrm{a}$, which is consistent with the expression of lipid/ cholesterol synthetic genes being androgen regulated, the cellular level of cholesterol was significantly increased by DHT treatment in CWR22-RV1 cells. Since cholesterol targeting has been shown to alter lipid composition and cell survival in PCa models, ${ }^{38}$ we treated cells with statins, an inhibitor class that blocks the activity of HMG-CoA reductase (the rate-limiting enzyme that regulates the metabolic pathway of cholesterol and other isoprenoids), to determine the impact of cholesterol/sterol biosynthesis on CRPC progression. To accomplish this, we first examined the effect of simvastatin, which has been previously shown to inhibit tumor growth in PCa models, ${ }^{38-40}$ on AR activity in CWR22-RV1 cells since cholesterol is the precursor for intratumoral steroid synthesis. As shown in Figure 5b, simvastatin did not significantly affect basal or androgen-induced AR activities, indicating that intratumoral androgen synthesis may not be essential for reactivating $A R$ signaling in AR-V-expressing CWR22-RV1 cells. Instead, simvastatin treatment greatly decreased the metabolic hub mTOR activity (based on phosphorylated S6), which may subsequently stabilize D-cyclins and stimulate G1/S progression of PCa cells ${ }^{25}$ (Figure $5 \mathrm{C}$ ). We then examined the effect of simvastatin on cellular proliferation and apoptosis. CWR22-RV1 is a CRPC cell line that can proliferate without androgen treatment (Figure $5 \mathrm{~d}$ ). Treating the cells with simvastatin suppressed cellular proliferation (Figure $5 \mathrm{~d}$ ) and induced apoptosis (Figure 5e). Finally, we treated CWR22-RV1derived CRPC xenografts (grown in castrated male mice) with short-term simvastatin and showed that this treatment significantly impaired the tumor growth (Figure 5f). Similar to the in vitro study, blocking cholesterol synthesis did not affect AR activity significantly but markedly blocked mTOR activity in these xenograft tumors (Figures $5 \mathrm{~g}$ and $\mathrm{h}$ ). Collectively, these results indicate that restoration of AR-mediated lipid synthesis is critical to drive CRPC recurrence through the activation of PI3K-mTOR pathways.

Although the expression of $A R-V s$ is generally undetectable or very low in LNCaP, many LNCaP-derived CRPC models express higher level of AR-Vs. ${ }^{18,34}$ Therefore, we further examined the effect of statin treatment in C4-2 cells (an LNCaP-derived CRPC cell line), where AR-V7 expression can be readily detected. Similar to RV1 cells, the expression of lipid synthetic genes was also DHTinduced in C4-2 cells (Figure 6a), suggesting that the AR regulation of lipid synthetic pathways are preserved and restored in this model, although multiple mechanisms in addition to expressing $A R-V 7$ may also contribute to the $A R$ reactivation in C4-2 cells. $^{21,41,42}$ Consistently, the mTOR activation was blocked by simvastatin treatment in C4-2 cells (Figure 6b). However, unlike in CWR22-RV1 cells the simvastatin treatment also decreased the basal AR activity in C4-2 cells (Figure 6c). This effect was presumably through blocking CYP11A1-dependent steroid synthesis, which was shown to be activated in C4-2 cells and produce progesterone to drive AR activity (LNCaP/C4-2 express T877 A mutant AR) in absence of androgen stimulation. ${ }^{21}$ Importantly, the statin treatment prevented the G1/S cell cycle progression of C4-2 cells cultured in either partial or completely hormone-depleted medium (Figures $6 \mathrm{~d}$ and e), indicating that the lipid biosynthesis is essential for the proliferation of C4-2 cells.

We next sought to determine the direct role of lipid synthesis genes in promoting CRPC growth. ACSL3 is a member of the longchain acyl-CoA synthetase that plays a key role in fatty acid metabolism and has been reported recently as an important driver for tumorigenesis of lung cancer with KRAS mutation. ${ }^{43}$ To determine if ACSL3 activity contributes to CRPC cell growth, we treated CWR22-RV1 cells with a specific inhibitor, Triacsin C, and compared its activity with statin treatment. As seen in Figure $7 \mathrm{a}$, low-dose treatments of Triacsin C (1-5 $\mu \mathrm{m})$ have the similar effect as simvastatin in decreasing cell proliferation ( 20\%), suggesting ACSL3 may play a role in CRPC cell growth. However, the $\mathrm{IC}_{50}$ of Triacsin $C$ treatment was significantly higher than simvastatin, indicating that this inhibitor may be less potent or inhibition of ACSL3 may not be as effective as inhibition of HMG-CoA reductase in suppressing CRPC cell proliferation. Next, we also assessed the clinical potential of targeting ELOVL7, a critical enzyme that mediates elongation of fatty acids, in a CRPC model. As shown in Figures $7 b$ and $c$, the ELOVL7 expression was greatly reduced by stable infection of shRNA against ELOVL7. We then examined whether silencing ELOVL7 has an impact on the development of 
a

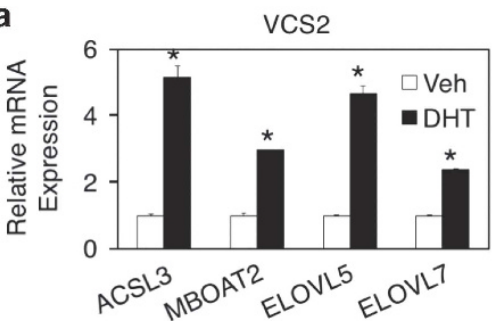

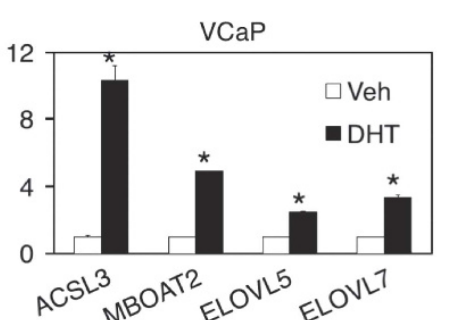

LNCaP

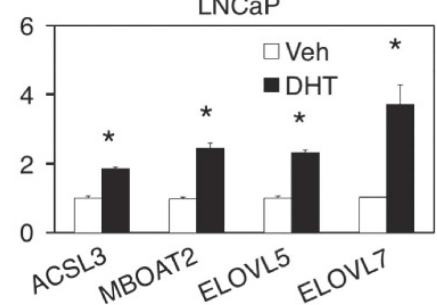

b

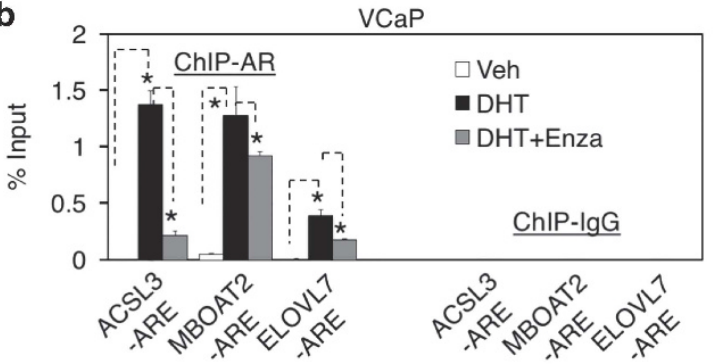

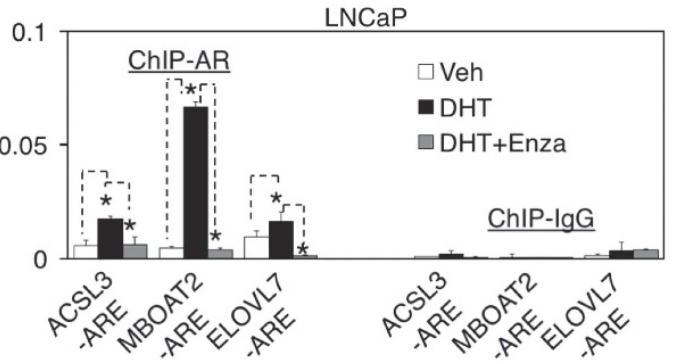

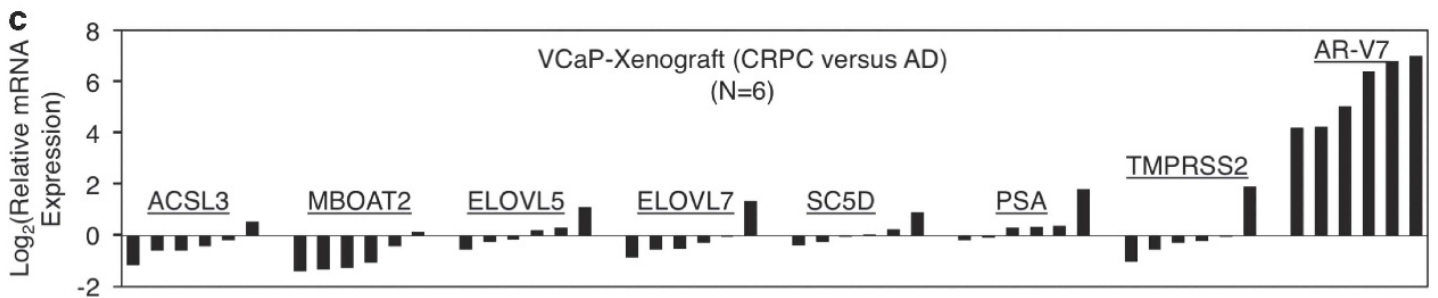

d
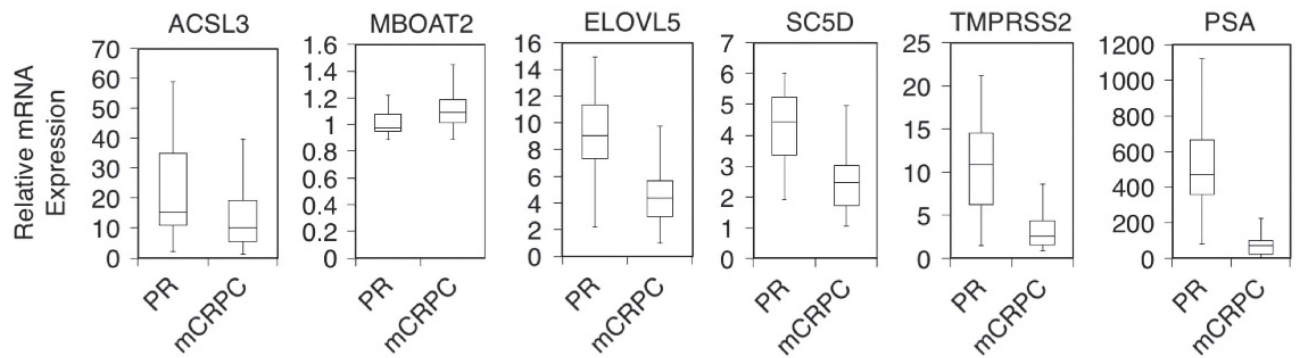

(Cai et al., Prostate)
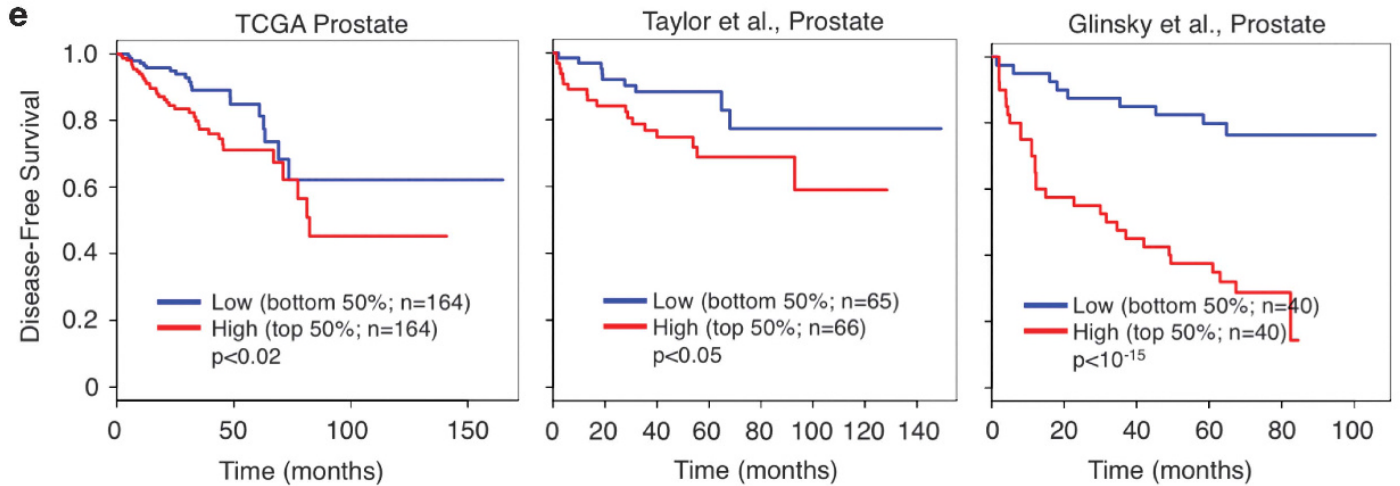

Figure 3. AR-mediated lipid biosynthesis is restored in CRPC. (a) qRT-PCT analysis for expression of indicated genes in VCS2, VCaP, LNCaP cells treated with vehicle or DHT (10 nM) for $24 \mathrm{~h}$. (b) ChIP-qPCR analysis for AR recruitment at indicated gene loci in VCaP and LNCaP cells treated by DHT alone ( $4 \mathrm{~h}$ ) versus DHT plus enzalutamide (pretreat $4 \mathrm{~h})$. (c) mRNA from tissue biopses of VCaP xenografts $(n=6)$ at pre-castration $(A D)$ and at relapse (CRPC) were extracted and subjected to qRT-PCR analysis. Gene expression is normalized to its level at pre-castration. (d) Box plot for expression of indicated genes in hormone-dependent primary PCa versus CRPC bone metastases (database: GSE32269). (e) KaplanMeier plotting was performed for the 32-gene subset of lipid synthesis genes on the TCGA, Taylor and Glinsky PCa cohorts. Blue curve represents bottom $50 \%$ patients with low expression and red curve represents top $50 \%$ patients with high expression. 
a

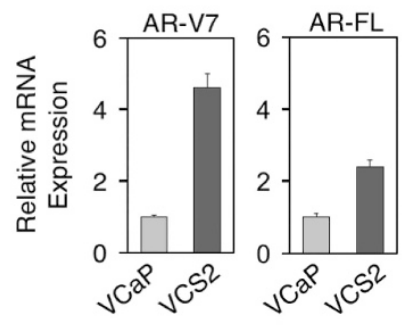

C

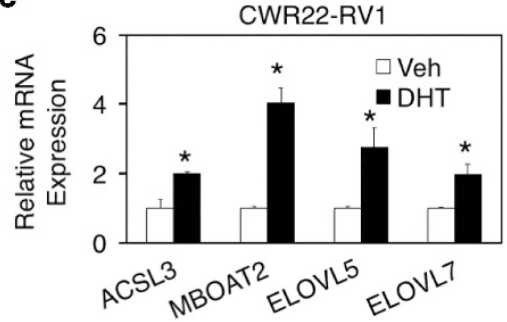

b

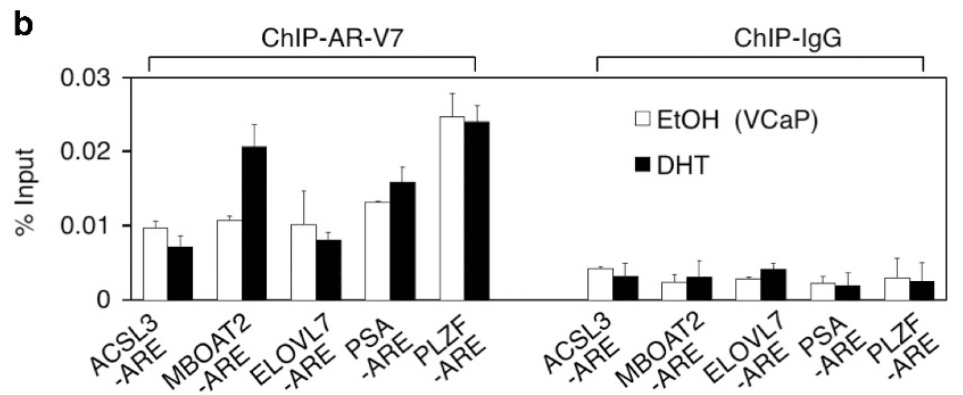

d

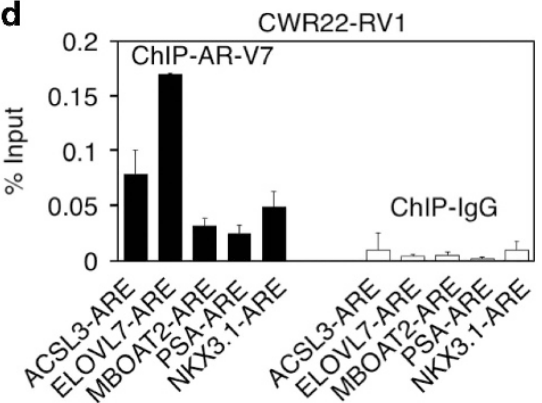

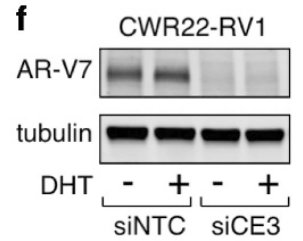

e

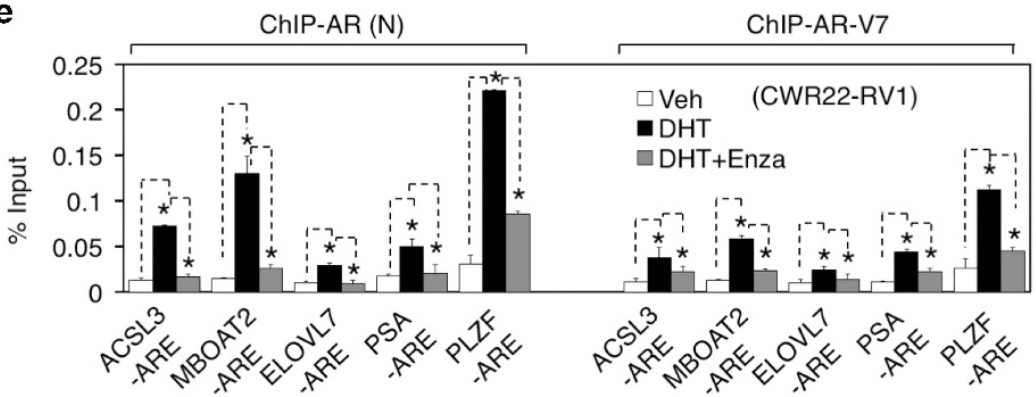

g
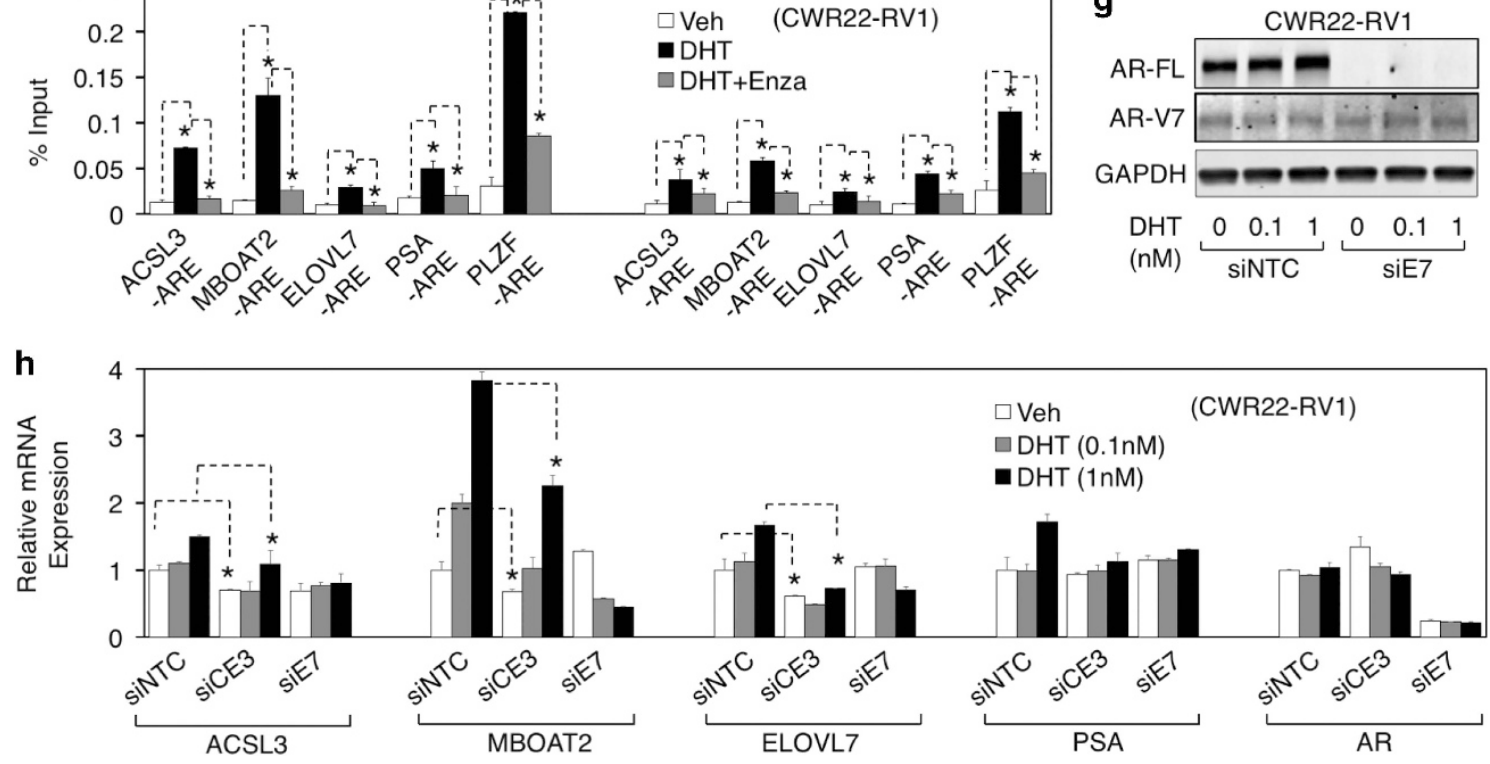

Figure 4. The expression of AR-V7 contributes to the reactivation of lipid synthesis in CRPC cells. (a) qRT-PCR analysis for AR-V7 in VCS2 and VCaP cells. (b) ChIP-qPCR analysis for AR-V7 recruitment using AR-V7 specific antibody at indicated gene loci in VCaP cells treated by DHT for 4hr. (c) qRT-PCR analysis for indicated genes in CWR22-RV1 cells treated with DHT for $24 \mathrm{~h}$. (d) ChIP-qPCR analysis for AR-V7 recruitment at indicated gene loci in CWR22-RV1 cells. (e) ChIP-qPCR analysis for total AR and AR-V7 recruitment at indicated gene loci in CWR22-RV1 cells treated by DHT alone (4hr) versus DHT plus enzalutamide (pretreat $4 \mathbf{h})$. (f-h) CWR22-RV1 cells were transfected with siNTC, siCE3, or siE7 for 2 days (d) in hormone-depleted medium and then treated with (f) 0-10 nM DHT or (g, h) 0-1 nM DHT for $24 \mathrm{~h}$, followed by (f), g) immunoblotting for AR-V7 or AR-FL (full-length) and by (H) qRT-PCR analysis of indicated genes.

CRPC xenograft tumors. As seen in Figure 7d, the growth of the xenograft tumors was significantly reduced by silencing ELOVL7 (Figure 7d), indicating an important role of fatty acid synthesis in driving CRPC progression. Overall, these in vitro and in vivo studies in CRPC models all support that AR-mediated lipid biosynthesis is essential for driving CRPC development.

\section{DISCUSSION}

$\mathrm{VCaP}$ is an androgen-sensitive PCa cell line that harbors the common TMPRSS2-ERG chromosomal alteration and $A R$ amplification. In this study, we have established a VCaP-derived CRPC model to assess the reprogramming of the AR cistrome. This xenograft-based model has many key human CRPC characteristics, including increased expression of full-length AR, AR splice variants, and androgen synthetic genes as well as restored $A R$ signaling. ${ }^{20-23}$ Interestingly, the results from ChIP-seq for total AR binding did not indicate significant alterations of the AR transcriptional program in this CRPC progression model, suggesting PCa cells can adapt to low androgen environment and reactivate AR signaling without triggering further AR-activation of other proliferation-related genes. This may seem to be 
a

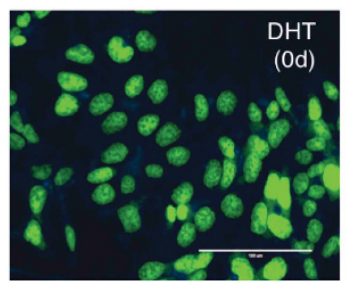

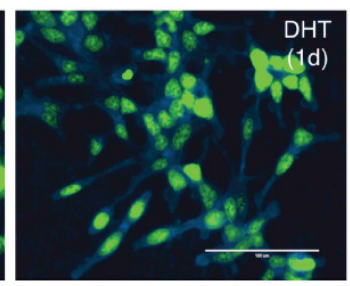

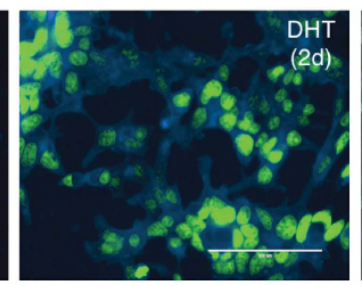

b

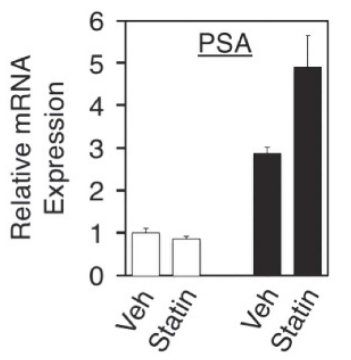

d
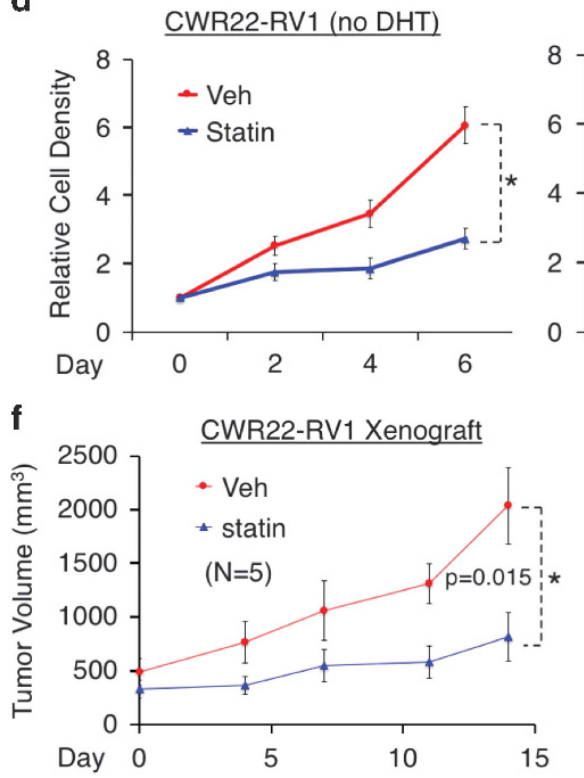
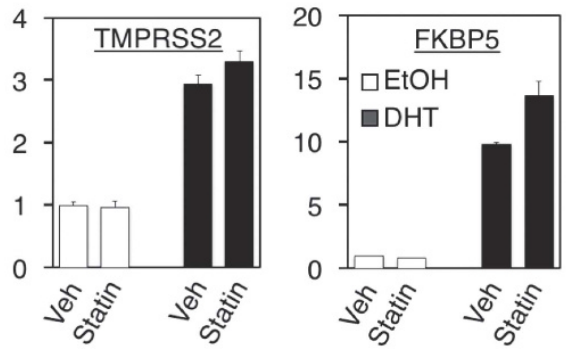

CWR22-RV1 (+10nM DHT)
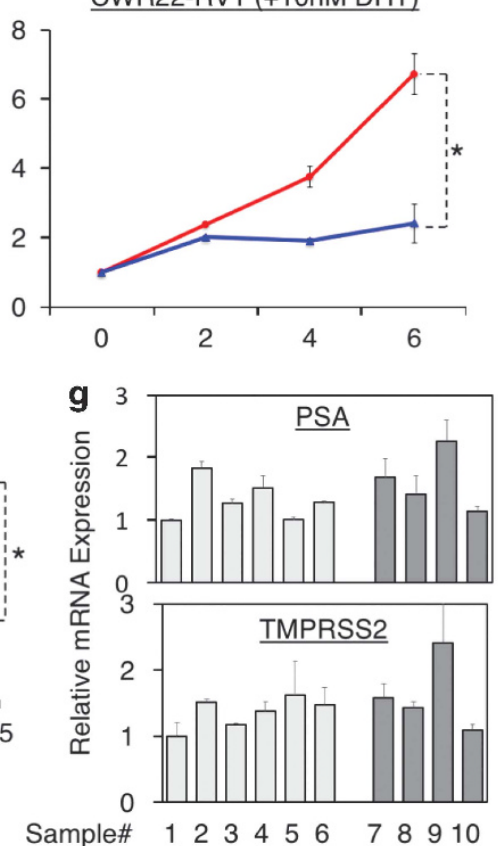

c

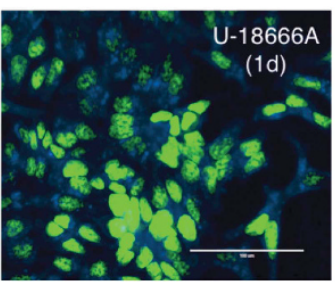

CWR22-RV1
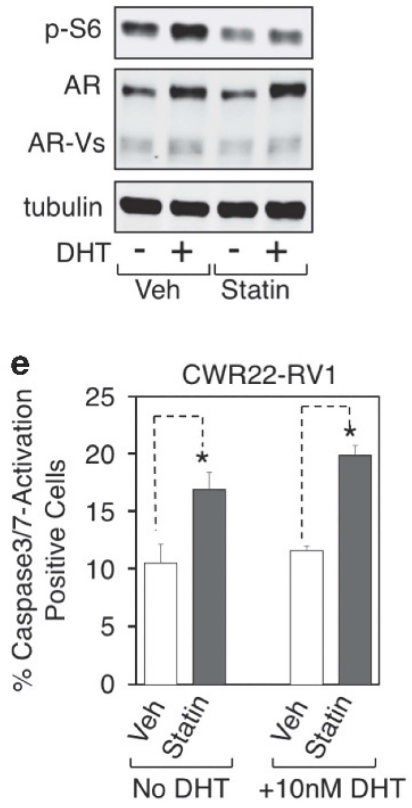

h

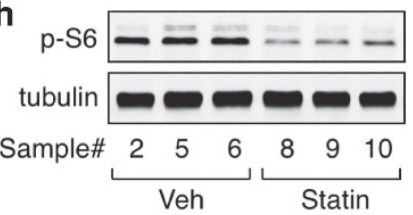

Figure 5. Blocking the lipid synthesis pathways suppresses tumor growth in the CWR22-RV1-derived CRPC xenograft model. (a) Immunofluorescence staining for cholesterol (blue) in CWR22-RV1 cells treated with DHT (10nM) for 1d, 2d and cholesterol trafficking inhibitor U-18666 A (1 $\mu \mathrm{m})$ for 1d (positive control). Nuclear compartment was determined by SYBR green staining (green). (b, c) CWR22-RV1 cells were treated with DHT alone and/or statin $(10 \mu \mathrm{m}$, pretreat $4 \mathrm{~h})$, followed by (b) qRT-PCR for indicated genes and (c) immunoblotting for Ser244-phosphorylated S6 and AR (using antibody against N-terminus of AR). (d, e) CWR22-RV1 cells were treated with statin and/or DHT, followed by flow cytometry cell counting of (d) live cells and (e) caspase-3/7 activity-high cells. (f-h) Castrated SCID mice (age $\sim 6$ weeks) bearing CWR22-RV1 xenograft tumors $(N=5)$ were treated with vehicle or statin $(10 \mathrm{mg} / \mathrm{kg}$ daily), followed by $(\mathbf{f})$ measuring tumor volume, (g) qRT-PCR analysis for mRNA expression from biopsies collected at day 15, and (h) immunoblotting for p-S6 in those samples. The statistical analysis was done using student's $t$-test and the sample size was estimated based on power analysis. The animals were randomized into two experimental groups and blinding was done for tumor measurement.

contradictory to the previous study showing that AR is reprogrammed to stimulate the expression of mitotic genes in the LNCaP-abl cell line model. ${ }^{12}$ However, our model may represent a distinct class of CRPC progressive tumors that may be specifically driven by increased expression of $A R-V s$. Another study using human PCa tissues also revealed significant reprogramming of $A R$ signaling in CRPC samples versus untreated primary tumor samples. ${ }^{13}$ However, this may be due to the selection of their primary PCa samples as the results indicate no substantial overlap of AR-binding sites between untreated PCa samples and PCa cell lines, while other studies have shown major overlapping. ${ }^{11}$ For
CRPC tumors that express high levels of $A R-V s$, we hypothesize that the restoration of AR activity can be achieved by dimerization of full-length $A R$ and $A R-V s$ that preferentially binds to the same AR-mediated enhancers at lipid synthetic genes. This hypothesis is supported by a previous report studying global binding of ARV7. ${ }^{44}$ The expression of $A R-V 7$ can be further increased in response to more intensive androgen-deprivation such as abiraterone or enzalutamide treatment, and its detection is associated with therapeutic resistance. ${ }^{19,23,36}$ Interestingly, our results are consistent with these studies, showing that increased lipid biosynthesis (potentially reactivated by AR-V7) was also associated with poor 
a

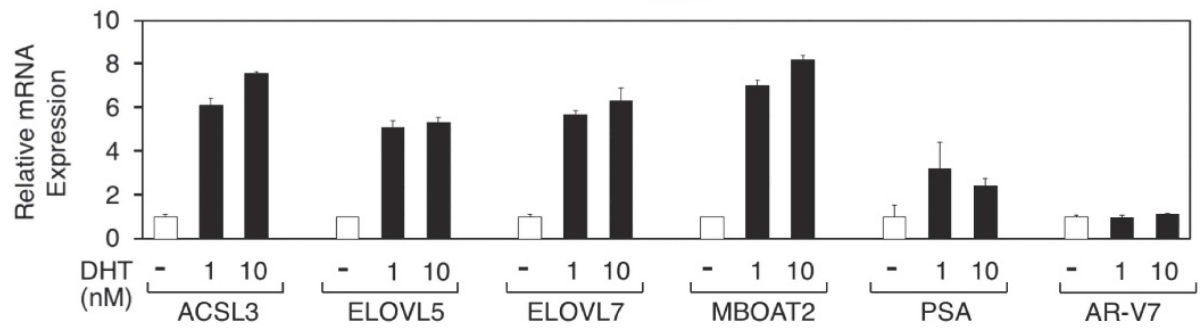

b
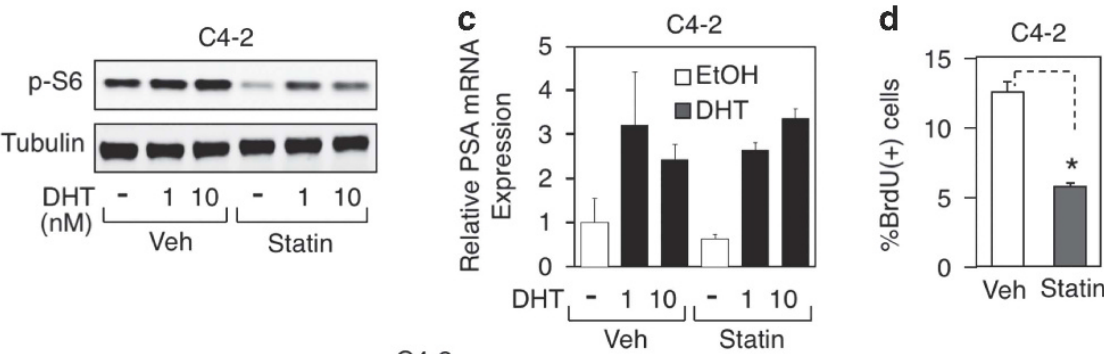

e

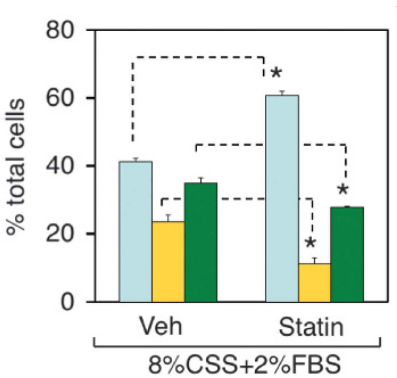

$\underline{\mathrm{C} 4-2}$

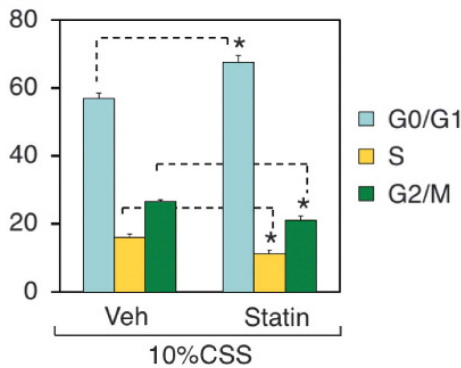

Figure 6. Blocking the lipid synthesis pathways prevented cell cycle progression in C4-2 CRPC cells. (a) qRT-PCR analysis for indicated genes in C4-2 cells treated with DHT for $24 \mathrm{~h}$. (b-d) C4-2 cells were treated with DHT alone and/or simvastatin (pretreat $4 \mathrm{~h}$ ) for $24 \mathrm{~h}$, followed by (b) immunoblotting for p-S6, (c) qRT-PCR for PSA mRNA expression, and (d) BrdU incorporation assay. (e) C4-2 cells grown in medium with 8\% CSS plus $2 \% \mathrm{FBS}$ or $10 \% \mathrm{CSS}$ were treated with simvastatin for $24 \mathrm{~h}$ and followed by flow cytometry cells cycle analysis.

outcome in multiple clinical cohorts (Figure 3e), indicating the AR$\mathrm{V}$-mediated reactivation of lipid biosynthesis may drive the disease recurrence. Nonetheless, in order to demonstrate the association of $A R-V$ expression with the level of lipid biosynthesis, future studies are warranted to assess AR cistrome in human CRPC samples with high expression of $A R$-Vs versus samples with low expression of $A R-V s$.

To target lipid biosynthesis pathways in CRPC, we used the HMG-CoA reductase inhibitors, or statins, to block the cholesterol synthesis. In PCa, cholesterol can serve as a precursor of steroid synthesis and therefore increase the production of intratumoral androgens. However, in the AR-V7-high CWR22-RV1 cells, we did not observe any significant effect of statin treatment on $A R$ signaling (Figures $5 \mathrm{~b}$ and $\mathrm{g}$ ). One possible explanation is that CWR22-RV1 cells may adapt to very low level of intracellular androgen synthesis that is sufficient to drive AR-V7-mediated reactivation of $A R$ signaling. Statins not only prevent the production of cholesterol but also block the formation of many intermediary lipid molecules, which can function to stimulate signaling pathways such as PI3K-AKT and mTOR. ${ }^{38,40}$ Indeed, we have observed inactivation of mTOR activity by statin treatment in CRPC models (Figures 5 and 6), indicating the major function of cholesterol synthesis in driving CRPC progression may be to stimulate PI3K/AKT-mTOR pathway, which maintains cancer cell survival and proliferation. Statin treatments have been used to treat hormone-dependent PCa in clinical trials, but patients largely did not benefit from such therapies. ${ }^{45}$ However, a recent report suggests that statins should be used at the time of initiation of ADTs as men taking statins had a longer time to progression than non-users. ${ }^{46}$ This result is consistent with our preclinical studies on CWR22-RV1 xenografts, showing that statins can delay the recurrence of CRPC (Figure 5f). As the castration in the mouse model (lack of CYP17 expression in adrenal) more aggressively reduces androgen levels than human ADT therapy, our study may suggest that statin treatment can improve the efficacy of more intensive ADTs such as abiraterone or enzalutamide treatment. Furthermore, in addition to statins, the inhibitors that block fatty acid synthesis and other lipid biosynthesis pathways (for example, targeting ACSL3 or ELOVLs, see Figure 7) need to be developed and further tested in conjunction with ADTs in preclinical CRPC models and in clinical trials. However, we speculate these combination treatment may be more effective in CRPC patients with higher expression of $A R-V s$.

In summary, our study demonstrates that PCa cells could progress to CRPC without significantly altering the transcriptional targets of AR. We further found that the genes mediating lipid biosynthesis pathways are the major targets of $A R$ that remain activated in CRPC. Mechanistically, we showed that the reactivation of lipid synthetic genes may be partially due to increased expression of $A R-V s$. Blocking cholesterol synthesis by statins decreased tumor growth in an AR-V7-expressing CRPC model by inactivating mTOR pathway. Silencing ELOVL7 expression also substantially reduced the growth of CRPC tumors. This study provides a strong rationale to assess the therapeutic efficacy of inhibitors that block lipid biosynthesis in combination with more aggressive ADTs to treat CRPC patients, particularly those with high expression of AR splice variants. 


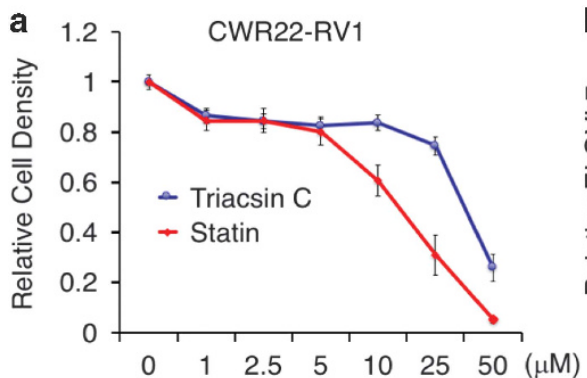

b

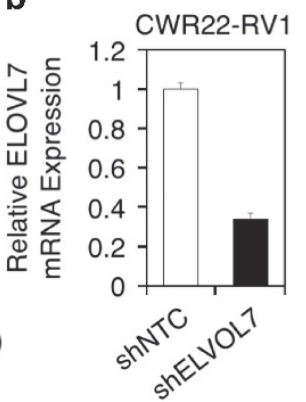

C

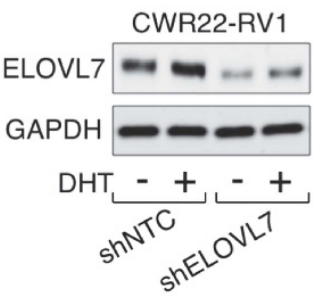

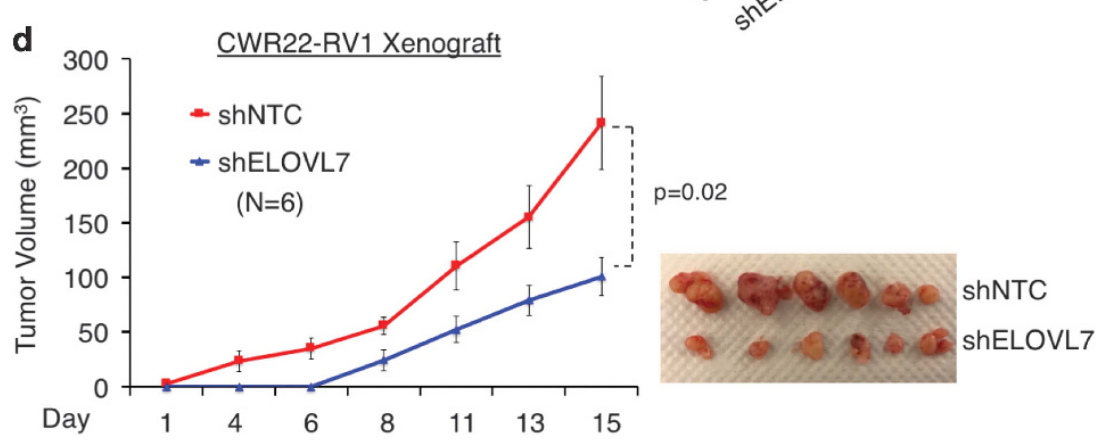

Figure 7. Targeting the fatty acid synthesis pathways reduces tumor growth in the CWR22-RV1-derived CRPC model. (a) CWR22-RV1 cells (in absence of androgen) were treated with statin or Triacsin C (purchased from Cayman Chemical) (0-50 $\mu \mathrm{m})$ for $6 \mathrm{~d}$, followed by flow cytometry cell counting. (b, c) CWR22-RV1 cells were stably infected by lenti-viral shRNA against ELOVL7 (shELOVL7, purchased from Dharmacon) or nontarget control (shNTC) with or without $10 \mathrm{~nm}$ DHT, followed by (b) qRT-PCR for ELOVL7 and (c) immunoblotting for ELOVL7. (d) CWR22-RV1 cells stably expressing shNTC or shELOVL7 were subcutaneously injected into castrated male SCID mice ( $N=6$, age $\sim 6$ weeks). The first measurement of tumor volume was at $\sim 2$ weeks after injection. The statistical analysis was done using student's $t$-test and the sample size was estimated based on power analysis. After the mice were sacrificed at $15 \mathrm{~d}$, the xenograft tumors were extracted and shown.

\section{MATERIALS AND METHODS}

Cell lines and cell culture

The VCaP, C4-2, and CWR22-RV1 cells were recently authenticated using short tandem repeat (STR) profiling by DDC Medical (Fairfield, OH, USA). VCS2 cells were derived from VCaP xenograft tumors and were cultured in RPMI-1640 with 2\% FBS (fetal bovine serum) and $8 \%$ CSS (charcoal-dextran stripped FBS serum) (Hyclone, Logan, UT, USA). VCaP cells were cultured in RPMI-1640 with 10\% FBS. CWR22-RV1 cells were cultured in RPMI-1640 with $10 \%$ CSS. C4-2 cells were cultured in RPMI- 1640 with $2 \%$ FBS plus $8 \%$ CSS. For androgen stimulation assays, cells were grown to $50-60 \%$ confluence in medium containing $5 \%$ CSS for 3 days and then treated with DHT or inhibitors for $24 \mathrm{~h}$.

\section{Chromatin immunoprecipitation (ChIP)}

VCaP or VCS2 cell were treated with $10 \mathrm{~nm}$ of DHT for $4 \mathrm{hr}$, followed by ChIP-Seq analyses as previously described. ${ }^{47}$ Briefly, ChIP was carried out using anti-AR antibody (Santa Cruz, Dallas, TX, USA, Sc-816×), followed by libraries construction and sequencing using the Illumina Genome Analyzer (San Diego, CA, USA). Significantly enriched regions $\left(P<10^{-15}\right.$ or $\left.<10^{-5}\right)$ were detected using the MACS software (2.0) with default parameters. The Gene Expression Omnibus (GEO) accession number for the ChIP-Seq analyses is GSE32345.

For preparation of ChIP-qPCR, dispensed cells were formalin fixed, lysed, and sonicated to break the chromatin into $500-800 \mathrm{bp}$ fragments, followed by immunoprecipitation. The GPCR was carried out using SYBR Green (Thermo Fisher Scientific, Waltham, MA, USA). The primers are listed as following: ACSL3-ARE: forward, 5'-GAGTTGTCATCCTGGGCACT-3', reverse, 5'-GGGGCCTGATTATTGGGTAT-3'; MBOAT2-ARE: forward, 5'-GTAGGTTGG ACTGGCAGCA-3', reverse, 5'-CGTAGCACCACGCATTACTC-3'; ELOVL7-ARE: forward, 5'-CATTGAACTTGAAGTACGCGTTAG-3', reverse, 5'-TाTGCTGTTGT GGATAGAACG-3'; PSA-ARE: forward, 5'-GCCTGGATCTGAGAGAGATATCATC -3', reverse, 5'-ACACCTIIIIIITTCTGGATTGTTG-3'; PLZF-ARE: forward, 5'-ACACCATGGCCTGTTGTAAA-3', reverse, 5'-ACCAAAACAGCAGACCCAAA -3'; NKX3.1-ARE: forward, 5'-CTGGCAAAGAGCATCTAGGG-3', reverse, 5'-GG CACTTCCTGAGCAAACTT-3'.

\section{RT-PCR and immunoblotting}

The mRNA was measured using real-time RT-PCR with Taqman one-step RT-PCR reagents and results were normalized to co-amplified GAPDH. The primers and probes are listed as following: ACSL3 (Hs00244853_m1), MBOAT2 (Hs01027245_m1), ELOVL5 (Hs01094711_m1), ELOVL7 (Hs00405151_m1), SC5D (Hs00999007_m1), NANS (Hs00219054_m1), TMPRSS2 (Hs01120965_m1), NKX3.1 (Hs00171834_m1) (purchased from Applied Biosystems, Foster City, CA, USA); PSA: forward, 5'-GATGA AACAGGCTGTGCCG-3', reverse, 5'-CCTCACAGCTACCCACTGCA-3', probe, 5'-FAM-CAGGAACAAAAGCGTGATCTTGCTGGG-3'; AR-V7: forward: 5'-CGGA AATGTTATGAAGCAGGGATGA-3', reverse, 5'-CTGGTCATITTGAGATGCTTGC AAT-3', probe, 5'-FAM-GGAGAAAAATTCCGGGT-3'. For immunoblotting, cells were lysed with RIPA buffer with protease inhibitors and antiSer240/244 phosphorylated S6 (Cell Signaling, Danvers, MA, USA), anti-AR (Millipore, Billerica, MA, USA), anti-AR-V7, anti-ELOVL7, anti- $\beta$-actin, antiGAPDH (Abcam, Cambridge, MA, USA), or anti- $\beta$-tubulin (Upstate, Billerica, $M A, U S A)$ antibodies were used. Gels shown are representative of at least 3 independent experiments.

\section{Flow cytometry}

After treatments, cells were collected and fixed with $70 \%$ ethanol for $3 \mathrm{~h}$, followed by staining with Muse Cell Cycle Assay kit (cell cycle), Count \& Viability Assay kit (proliferation), or Caspase-3/7 Assay kit (apoptosis) for 30 min and cell counting by Muse Cell Analyzer (EMD Millipore, Billerica, MA, USA). For BrdU incorporation assay, C4-2 cells grown in 5\%CSS were incubated with BrdU for indicated period, followed by fixation and permeabilization. After re-fixation, cells were treat with DNase to expose incorporated BrdU, followed by staining BrdU with fluorescent antibody and FACS analysis.

\section{Cholesterol staining}

CWR22-RV1 cells were seeded with 10\%CSS in Nunc Lab-Tek II chamber slides (Thermo Fisher Scientific), and treated by DHT (10 nM) for 1 and 2 days, or $\mathrm{U}-18666 \mathrm{~A}(1 \mu \mathrm{m})$ for 1 day. The cells were then fixed for $10 \mathrm{~min}$ and then treated with Filipin III solution for 30 min using the Cholesterol Assay Kit (Abcam). SYBR green staining (Invitrogen, Carlsbad, CA, USA) was 
performed for $5 \mathrm{~min}$ and images were taken under fluorescence microscope.

\section{CWR22-RV1 xenografts}

Xenograft establishment has been previously described. ${ }^{22}$ Tumor volume was measured by manual caliper.

\section{Statistical analysis}

Data in bar graphs represent mean \pm s.d. of at least 3 biological repeats. Statistical analysis was performed using Student's $t$-test by comparing treatment versus vehicle control or otherwise as indicated. ${ }^{*} P<0.05$ was considered to be statistically significant. The results for immunoblotting are representative of at least three experiments.

\section{CONFLICT OF INTEREST}

The authors declare no conflict of interest.

\section{ACKNOWLEDGEMENTS}

This work is supported by grants from NIH (R00 CA166507 to CC, and SPORE in Prostate Cancer P50 CA090381-13), DOD (W81XWH-15-1-0554 to SG, and W81XWH-15-1-0519 and W81XWH-16-1-0445 to CC), CIHR (142246 to HHH), Prostate Cancer Canada (RS2016-1022 to HHH), NSERC (498706 to HHH), CCSRI (703800 to $\mathrm{HHH}$ ) and Princess Margaret Cancer Foundation (to $\mathrm{HHH}$ ). We thank Dr Steven P Balk (Beth Israel Deaconess Medical Center) for the advice of experiment design and Drs Myles Brown and X Shirley Liu (Dana-Farber Cancer Institute) for the support of bioinformatical analyses.

\section{REFERENCES}

1 Green SM, Mostaghel EA, Nelson PS. Androgen action and metabolism in prostate cancer. Mol Cell Endocrinol 2012; 360: 3-13.

2 Yuan X, Cai C, Chen S, Chen S, Yu Z, Balk SP. Androgen receptor functions in castration-resistant prostate cancer and mechanisms of resistance to new agents targeting the androgen axis. Oncogene 2014; 33: 2815-2825.

3 de Bono JS, Logothetis CJ, Molina A, Fizazi K, North S, Chu L et al. Abiraterone and increased survival in metastatic prostate cancer. N Engl J Med 2011; 364: 1995-2005.

4 Scher HI, Fizazi K, Saad F, Taplin ME, Sternberg CN, Miller K et al. Increased survival with enzalutamide in prostate cancer after chemotherapy. N Engl J Med 2012; 367: 1187-1197.

5 Ryan CJ, Molina A, Griffin T. Abiraterone in metastatic prostate cancer. N Engl J Med 2013; 368: 1458-1459.

6 Tomlins SA, Rhodes DR, Perner S, Dhanasekaran SM, Mehra R, Sun XW et al. Recurrent fusion of TMPRSS2 and ETS transcription factor genes in prostate cancer. Science 2005; 310: 644-648.

7 Yu J, Yu J, Mani RS, Cao Q, Brenner CJ, Cao X et al. An integrated network of androgen receptor, polycomb, and TMPRSS2-ERG gene fusions in prostate cancer progression. Cancer Cell 2010; 17: 443-454.

8 Cai C, Wang H, He HH, Chen S, He L, Ma F et al. ERG induces androgen receptormediated regulation of SOX9 in prostate cancer. J Clin Invest 2013; 123: 1109-1122.

9 Chen Y, Chi P, Rockowitz S, laquinta PJ, Shamu T, Shukla S et al. ETS factors reprogram the androgen receptor cistrome and prime prostate tumorigenesis in response to PTEN loss. Nat Med 2013; 19: 1023-1029.

10 Mills IG. Maintaining and reprogramming genomic androgen receptor activity in prostate cancer. Nat Rev Cancer 2014; 14: 187-198.

11 Pomerantz MM, Li F, Takeda DY, Lenci R, Chonkar A, Chabot M et al. The androgen receptor cistrome is extensively reprogrammed in human prostate tumorigenesis. Nat Genet 2015; 47: 1346-1351.

12 Wang Q, Li W, Zhang Y, Yuan X, Xu K, Yu J et al. Androgen receptor regulates a distinct transcription program in androgen-independent prostate cancer. Cell 2009; 138: 245-256.

13 Sharma NL, Massie CE, Ramos-Montoya A, Zecchini V, Scott HE, Lamb AD et al. The androgen receptor induces a distinct transcriptional program in castrationresistant prostate cancer in man. Cancer Cell 2013; 23: 35-47.

14 Dehm SM, Schmidt LJ, Heemers HV, Vessella RL, Tindall DJ. Splicing of a novel androgen receptor exon generates a constitutively active androgen receptor that mediates prostate cancer therapy resistance. Cancer Res 2008; 68: 5469-5477.

15 Guo Z, Yang X, Sun F, Jiang R, Linn DE, Chen $\mathrm{H}$ et al. A novel androgen receptor splice variant is up-regulated during prostate cancer progression and promotes androgen depletion-resistant growth. Cancer Res 2009; 69: 2305-2313.
16 Hu R, Dunn TA, Wei S, Isharwal S, Veltri RW, Humphreys E et al. Ligandindependent androgen receptor variants derived from splicing of cryptic exons signify hormone-refractory prostate cancer. Cancer Res 2009; 69: 16-22.

17 Dehm SM, Tindall DJ. Alternatively spliced androgen receptor variants. Endocr Relat Cancer 2011; 18: R183-R196.

18 Li Y, Chan SC, Brand LJ, Hwang TH, Silverstein KA, Dehm SM. Androgen receptor splice variants mediate enzalutamide resistance in castration-resistant prostate cancer cell lines. Cancer Res 2013; 73: 483-489.

19 Antonarakis ES, Lu C, Wang H, Luber B, Nakazawa M, Roeser JC et al. AR-V7 and resistance to enzalutamide and abiraterone in prostate cancer. N Engl J Med 2014; 371: 1028-1038.

20 Cai C, Wang H, Xu Y, Chen S, Balk SP. Reactivation of androgen receptor-regulated TMPRSS2:ERG gene expression in castration-resistant prostate cancer. Cancer Res 2009; 69: 6027-6032.

21 Cai C, Chen S, Ng P, Bubley GJ, Nelson PS, Mostaghel EA et al. Intratumoral de novo steroid synthesis activates androgen receptor in castration-resistant prostate cancer and is upregulated by treatment with CYP17A1 inhibitors. Cancer Res 2011; 71: 6503-6513.

22 Cai $\mathrm{C}$, He HH, Chen S, Coleman I, Wang H, Fang Z et al. Androgen receptor gene expression in prostate cancer is directly suppressed by the androgen receptor through recruitment of lysine-specific demethylase 1. Cancer Cell 2011; 20: 457-471

23 Yu Z, Chen S, Sowalsky AG, Voznesensky OS, Mostaghel EA, Nelson PS et al. Rapid induction of androgen receptor splice variants by androgen deprivation in prostate cancer. Clin Cancer Res 2014; 20: 1590-1600.

24 Massie CE, Lynch A, Ramos-Montoya A, Boren J, Stark R, Fazli L et al. The androgen receptor fuels prostate cancer by regulating central metabolism and biosynthesis. EMBO J 2011; 30: 2719-2733.

$25 \mathrm{Xu}$ Y, Chen SY, Ross KN, Balk SP. Androgens induce prostate cancer cell proliferation through mammalian target of rapamycin activation and posttranscriptional increases in cyclin D proteins. Cancer Res 2006; 66: 7783-7792.

26 Evans AJ. Alpha-methylacyl CoA racemase (P504S): overview and potential uses in diagnostic pathology as applied to prostate needle biopsies. J Clin Pathol 2003; 56: 892-897.

27 Ananthanarayanan V, Deaton RJ, Yang XJ, Pins MR, Gann PH. Alpha-methylacylCoA racemase (AMACR) expression in normal prostatic glands and high-grade prostatic intraepithelial neoplasia (HGPIN): association with diagnosis of prostate cancer. Prostate 2005; 63: 341-346

28 Migita T, Ruiz S, Fornari A, Fiorentino M, Priolo C, Zadra G et al. Fatty acid synthase: a metabolic enzyme and candidate oncogene in prostate cancer. J Nat/ Cancer Inst 2009; 101: 519-532.

29 Tamura K, Makino A, Hullin-Matsuda F, Kobayashi T, Furihata M, Chung S et al. Novel lipogenic enzyme ELOVL7 is involved in prostate cancer growth through saturated long-chain fatty acid metabolism. Cancer Res 2009; 69: 8133-8140.

30 Stanbrough M, Bubley GJ, Ross K, Golub TR, Rubin MA, Penning TM et al. Increased expression of genes converting adrenal androgens to testosterone in androgen-independent prostate cancer. Cancer Res 2006; 66: 2815-2825.

31 Glinsky GV, Glinskii AB, Stephenson AJ, Hoffman RM, Gerald WL. Gene expression profiling predicts clinical outcome of prostate cancer. J Clin Invest 2004; 113: 913-923.

32 Taylor BS, Schultz N, Hieronymus H, Gopalan A, Xiao Y, Carver BS et al. Integrative genomic profiling of human prostate cancer. Cancer Cell 2010; 18: 11-22.

33 Cancer Genome Atlas Research N. The molecular taxonomy of primary prostate cancer. Cell 2015; 163: 1011-1025.

$34 \mathrm{Lu} \mathrm{C}$, Luo J. Decoding the androgen receptor splice variants. Transl Androl Urol 2013; 2: 178-186.

35 Watson PA, Chen YF, Balbas MD, Wongvipat J, Socci ND, Viale A et al. Constitutively active androgen receptor splice variants expressed in castrationresistant prostate cancer require full-length androgen receptor. Proc Natl Acad Sci USA 2010; 107: 16759-16765.

36 Liu LL, Xie N, Sun S, Plymate S, Mostaghel E, Dong X. Mechanisms of the androgen receptor splicing in prostate cancer cells. Oncogene 2014; 33: 3140-3150.

37 Li Y, Alsagabi M, Fan D, Bova GS, Tewfik AH, Dehm SM. Intragenic rearrangement and altered RNA splicing of the androgen receptor in a cell-based model of prostate cancer progression. Cancer Res 2011; 71: 2108-2117.

38 Zhuang L, Kim J, Adam RM, Solomon KR, Freeman MR. Cholesterol targeting alters lipid raft composition and cell survival in prostate cancer cells and xenografts. J Clin Invest 2005; 115: 959-968.

39 Sivaprasad U, Abbas T, Dutta A. Differential efficacy of 3-hydroxy-3-methylglutaryl CoA reductase inhibitors on the cell cycle of prostate cancer cells. Mol Cancer Ther 2006; 5: 2310-2316.

40 Kochuparambil ST, Al-Husein B, Goc A, Soliman S, Somanath PR. Anticancer efficacy of simvastatin on prostate cancer cells and tumor xenografts is associated with inhibition of Akt and reduced prostate-specific antigen expression. J Pharmacol Exp Ther 2011; 336: 496-505. 
41 Chen S, Xu Y, Yuan X, Bubley GJ, Balk SP. Androgen receptor phosphorylation and stabilization in prostate cancer by cyclin-dependent kinase 1. Proc Natl Acad Sci USA 2006; 103: 15969-15974.

42 Dehm SM, Tindall DJ. Ligand-independent androgen receptor activity is activation function-2-independent and resistant to antiandrogens in androgen refractory prostate cancer cells. J Biol Chem 2006; 281: 27882-27893.

43 Padanad MS, Konstantinidou G, Venkateswaran N, Melegari M, Rindhe S, Mitsche M et al. Fatty Acid oxidation mediated by acyl-coa synthetase long chain 3 is required for mutant kras lung tumorigenesis. Cell Rep 2016; 16: 1614-1628.

44 Chan SC, Selth LA, Li Y, Nyquist MD, Miao L, Bradner JE et al. Targeting chromatin binding regulation of constitutively active $A R$ variants to overcome prostate cancer resistance to endocrine-based therapies. Nucleic Acids Res 2015; 43: 5880-5897.

45 Pon D, Abe A, Gupta EK. A review of statin use and prostate cancer. Curr Atheroscler Rep 2015; 17: 474.

46 Harshman LC, Wang X, Nakabayashi M, Xie W, Valenca L, Werner L et al. Statin use at the time of initiation of androgen deprivation therapy and time to progression in patients with hormone-sensitive prostate cancer. JAMA Oncol 2015; 1: 495-504.

47 He HH, Meyer CA, Shin H, Bailey ST, Wei G, Wang Q et al. Nucleosome dynamics define transcriptional enhancers. Nat Genet 2010; 42: 343-347.

(c) (1) (2) This work is licensed under a Creative Commons AttributionConCommercial-ShareAlike 4.0 International License. The images or other third party material in this article are included in the article's Creative Commons license, unless indicated otherwise in the credit line; if the material is not included under the Creative Commons license, users will need to obtain permission from the license holder to reproduce the material. To view a copy of this license, visit http:// creativecommons.org/licenses/by-nc-sa/4.0/

(c) The Author(s) 2018 